\title{
AN INVESTIGATION OF AUTOMATIC CHANGE DETECTION FOR TOPOGRAPHIC MAP UPDATING
}

\author{
Patricia Duncan ${ }^{1} \&$ Julian Smit ${ }^{2}$ \\ ${ }^{1}$ The Chief Directorate: National Geospatial Information, Department of Rural Development and Land Reform, Cape Town, \\ South Africa \\ pduncan@ruraldevelopment.gov.za
}

${ }^{2}$ Geomatics Division, School of Architecture, Planning \& Geomatics, University of Cape Town, Cape Town, South Africa Julian.Smit@uct.ac.za

KEY WORDS: Mapping, Change Detection, Classification, Pixel, Object

\begin{abstract}
Changes to the landscape are constantly occurring and it is essential for geospatial and mapping organisations that these changes are regularly detected and captured, so that map databases can be updated to reflect the current status of the landscape. The Chief Directorate of National Geospatial Information (CD: NGI), South Africa's national mapping agency, currently relies on manual methods of detecting changes and capturing these changes. These manual methods are time consuming and labour intensive, and rely on the skills and interpretation of the operator. It is therefore necessary to move towards more automated methods in the production process at CD: NGI. The aim of this research is to do an investigation into a methodology for automatic or semi-automatic change detection for the purpose of updating topographic databases. The method investigated for detecting changes is through image classification as well as spatial analysis and is focussed on urban landscapes. The major data input into this study is high resolution aerial imagery and existing topographic vector data. Initial results indicate the traditional pixel-based image classification approaches are unsatisfactory for large scale land-use mapping and that object-orientated approaches hold more promise. Even in the instance of object-oriented image classification generalization of techniques on a broad-scale has provided inconsistent results. A solution may lie with a hybrid approach of pixel and object-oriented techniques.
\end{abstract}

\section{INTRODUCTION}

The Chief Directorate of National Geo-spatial Information (CD: NGI), South Africa's national mapping agency, is responsible for the official, definitive, national topographic mapping, aerial imagery and control survey network of South Africa. One of the responsibilities of the CD: NGI is the capturing and revision of topographical data into the national integrated database of geo-spatial information. The process of detecting changes to the landscape and updating CD: NGI's topographic database is currently performed manually, which is time consuming and relies on the knowledge and interpretation of the operator.

The focus of this research is on updating topographic data for urban built-up areas, as these areas can change rapidly. An automated method of detecting changes to these areas is needed so that the topographic database can be updated regularly. The proposed method of detecting change is through image classification. In this paper we will compare various methods of image classification for the purpose of updating topographic databases. The change detection part of the research will come at a later stage once the most appropriate method of image classification has been decided on. It is envisaged that changes will be detected by comparing the newly classified data with the existing topographic vector data.

The imagery used in this study is $0.5 \mathrm{~m}$ resolution aerial imagery. Available image bands are red, green, blue and near-infrared. Existing vector data representing topographical features is the basis for measuring and comparing changes that are detected.

\section{UPDATING TOPOGRAPHIC DATABASES THROUGH IMAGE CLASSIFICATION}

\section{PIXEL-BASED CLASSIFICATION}

\section{Supervised classification}

Using the maximum likelihood classification method, Walter \& Fritsch (1998) found that forests are recognised as homogenous and are well detected, while agricultural areas may show inconsistencies due to planting structure, but they could also be well detected. The water class was the most easily detected. Larger streets are recognised without significant problems, but sometimes there is confusion between pixels from the street class and pixels that represent house roofs due to their similar spectral characteristics. Pixels are only recognised as settlement areas if they represent house roofs, while other pixels in 
the settlement class are classified as other features such as forest or agricultural areas, due to the high resolution of the imagery and wide variety of building roof spectral properties. When tested with lower resolution imagery, settlements are recognised as uniform but the accuracy of the results deteriorates.

Classes must be decided on beforehand and adequate samples that represent the classes must be collected. For most super classes there will be subclasses for a feature due to the spectral variation within classes. For example, many samples of the class 'water' were collected as subclasses and merged to make the final water class. A further challenge lies in the fact that a class may consist of various land cover types which are spectrally diverse, but need to be grouped together. Such an example is the urban built-up class which may consist of buildings, gardens (vegetation), swimming pools and bare ground. One may consider classifying buildings separately, but this decision is influenced by the purpose of the classification, and in this case the built-up area was required. Even individual buildings can have a multitude of different land cover types and colours; for example, roof tiles, thatch, metal sheeting, etc. are all spectrally diverse.

In this example the maximum likelihood classification method was tested. Final classes consisted of water, vegetation, road, built-up and bare ground or sand.

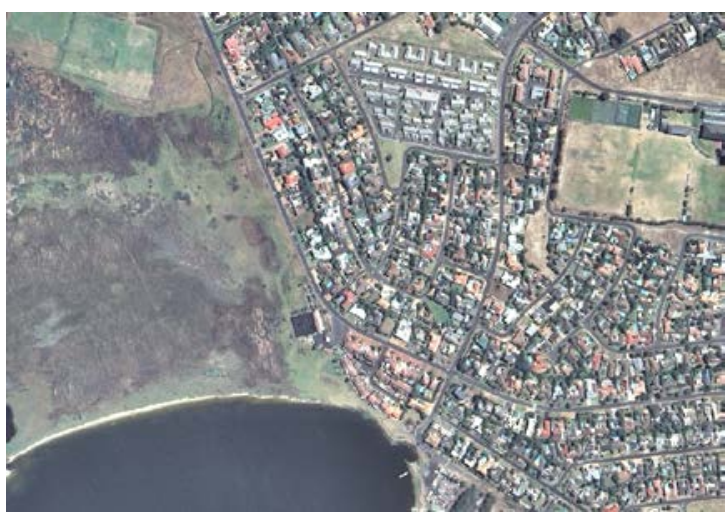

Figure 1: Subset of aerial image used in all classification methods tested

\begin{tabular}{|l|c|c|c|}
\hline Class name & $\begin{array}{l}\text { Producers } \\
\text { accuracy }\end{array}$ & $\begin{array}{l}\text { Users } \\
\text { accuracy }\end{array}$ & $\begin{array}{l}\text { KIA per } \\
\text { class }\end{array}$ \\
\hline Road & 0.67 & 0.40 & 0.34 \\
\hline $\begin{array}{l}\text { Bare ground } \\
\text { or sand }\end{array}$ & 1.00 & 1.00 & 1.00 \\
\hline Water & 0.74 & 1.00 & 1.00 \\
\hline Built-up & 0.73 & 0.66 & 0.43 \\
\hline Vegetation & 0.50 & 0.60 & 0.51 \\
\hline Overall accuracy & \multicolumn{2}{|c|}{$\mathbf{0 . 7 0}$} \\
\hline KIA & & \multicolumn{2}{|c|}{} \\
\hline
\end{tabular}

Table 1: Accuracy assessment and kappa statistics for pixel-based supervised method (Maximum Likelihood Classification)

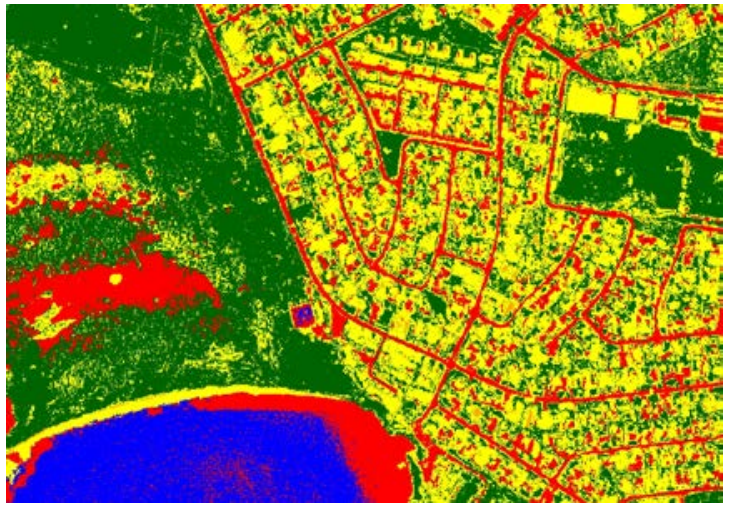

Figure 2: Results of supervised classification - maximum likelihood classification

The classification results indicate that there is overlap between the road and the built-up classes due to their spectral similarity. Some buildings were also incorrectly classified as bare ground or sand and some vegetation was incorrectly classified as roads and built-up areas.

\section{Unsupervised classification}

In unsupervised classification, pixel values within a certain land cover type should be close together in the measurement (spectral) space, whereas data in different classes should be reasonably well separated. The classes that result from unsupervised classification are spectral classes (Lillesand et al. 2004).

The unsupervised ISODATA method is popular in the classification of heterogeneous high resolution images as it is very successful in finding the spectral clusters that are inherent in images (Zhang 2001). Unsupervised classification may address some of the shortcomings of applying supervised classification for land use or land cover classification where classes have a high degree of spectral variability. Where there is a high degree of spectral variability, suitable training sites for relevant land use or land cover classes will always be difficult to achieve.

The unsupervised approach is simple and no training data or samples are needed, thus making it much faster to implement than the supervised approach. Another advantage is that the unsupervised classifier identifies the different spectral classes present in an image, which might not be obvious to an analyst applying a supervised classifier. Similarly, there may be so many spectral classes in a scene that it would be difficult to train on all of them. Since unsupervised classification is the identification of spectrally distinct classes in an image, the analyst must still use reference data to associate spectral classes with the land cover types of interest. The spectral classes identified may not be uniquely associated with a land cover type, and one may have several spectral classes representing a single feature class (Lillesand et al. 2004).

The unsupervised approach was tested using the ISODATA method. The results were not satisfactory and classes were not easily separated due to the large variability within individual classes. The accuracy of the 
unsupervised method was based on a visual inspection. Where there are many classes, there is the problem of a class being split into more than one class due to the spectral differences within a class. Where there are only a few classes, there is the problem of unrelated classes being classified as the same class.

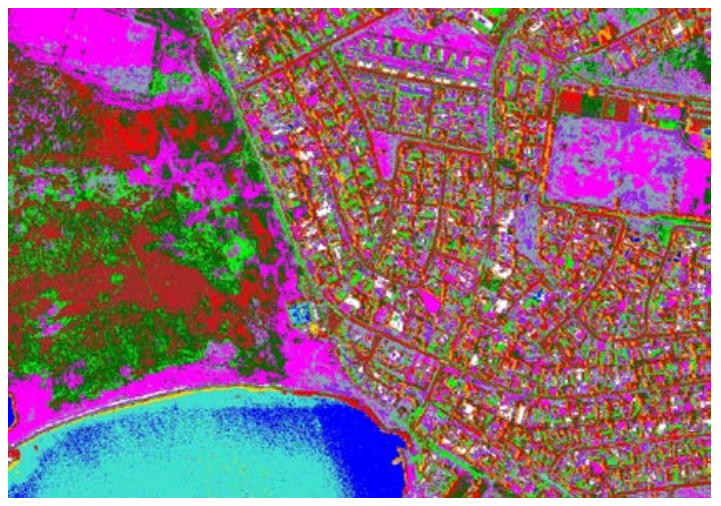

Figure 3: Results of unsupervised classification ISODATA method - 12 classes

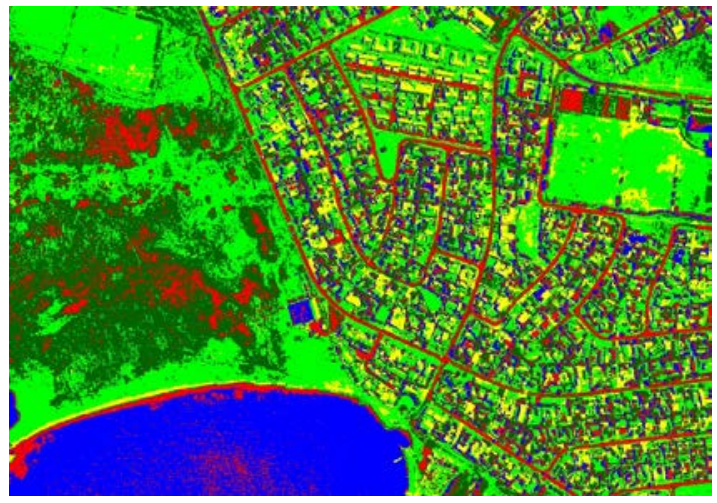

Figure 4: Results of unsupervised classification ISODATA method -5 classes

It is thus concluded that the pixel-based approach is not acceptable for classifying complex urban environments with very high resolution remote sensing data. The reasons for this are as follows (Hurskainen \& Pellikka 2004):

- Pixels do not sample the urban environment at the spatial scale to be mapped

- Building are represented by groups of pixels which should be treated as individual objects

- Buildings produce a wide range of spectral signatures

- Many features in the urban environment appear spectrally similar

\section{OBJECT-BASED CLASSIFICATION}

The limitation of the pixel in tackling issues of location, scale and distance has caused a shift towards objectbased classification (De Dapper et al. 2006). Even though traditional pixel-based classifiers are well developed and there are sophisticated variations, they do not make use of available spatial concepts. The need for context-based algorithms and object-oriented image processing is increasing and it is hypothesized that object-based image analysis will initiate new developments towards integrating GIS and remote sensing functions (Blaschke et al. 2000).

The software used for object-based classification in this study is eCognition. A necessary prerequisite for objectbased image classification is image segmentation. The shape of segments derived in eCognition is determined by the following parameters (Hofmann 2001):

- Weight of image channels: specify the weight of each spectral band in the segmentation. Channels with higher weights have a greater influence on object generation.

- $\quad$ Scale parameter: influences the average object size. This parameter determines the maximum allowed heterogeneity of the objects. The larger the scale parameter, the larger the objects become.

- Colour/Shape: the influence of colour vs. shape can be adjusted. The higher the shape value, the less spectral homogeneity influences the object generation.

- Smoothness/Compactness: These are attributes of the "shape" criterion. If the shape criterion is larger than 0 , the user can determine whether objects shall be more compact or more smooth.

- Level: determines whether a new generated image level will either overwrite a current level or whether the generated objects shall contain sub or super objects of an existing level. The order of generating the levels affects the objects' shape (top-down vs. bottom-up segmentation).

\section{Using spectral information for image segmentation}

In the first strategy, the image was segmented using the multiresolution segmentation algorithm in eCognition. All four image layers (red, green, blue and NIR) were used with equal weighing in the segmentation process. The size of segments was decided on by trial and error. Smaller segments were merged to create larger segments that consisted of built-up areas as opposed to individual buildings. These built-up areas consisted of residential buildings, gardens, roads, etc. It was difficult to obtain suitable segments using only spectral information. The segments were not uniform in shape and size, and some contained a mixture of classes that was not ideal. Some segments appeared homogenous in nature, but did not logically represent features in an image.

In the Figure $5 \mathrm{a}$, it can be seen that the selected segment contains a building, a portion of a road and some trees. These segments were created from initially smaller segments with scale parameter of 50 (Figure 5b), which were then used as the input into a multiresolution segmentation to create segments with a scale parameter of 100 (as seen in Figure 5a). An initial segmentation of 100 results in slightly different segments as can be seen in Figure 5c. 


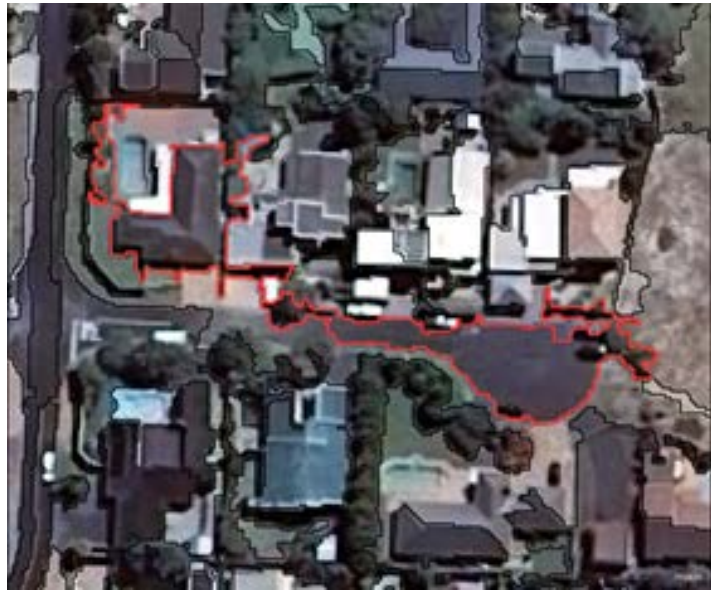

Figure 5a: Image segmentation (Level 2, scale: 100)

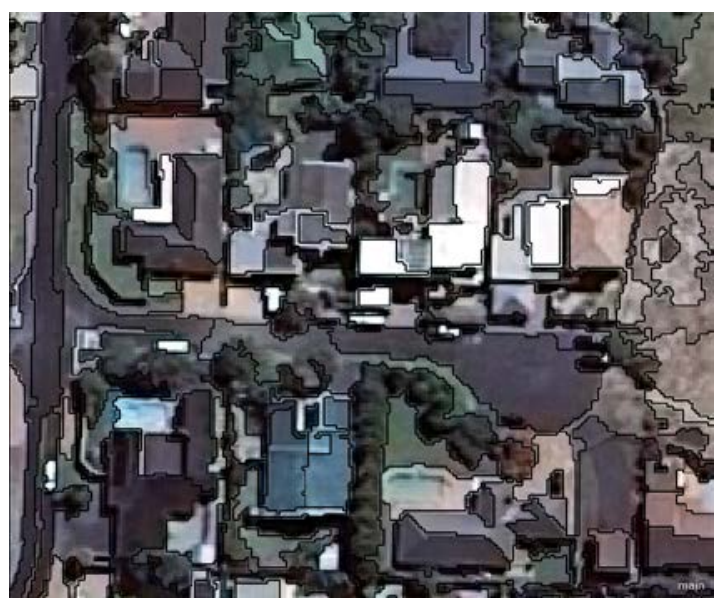

Figure 5b: Image segmentation (Level 1, scale: 50)

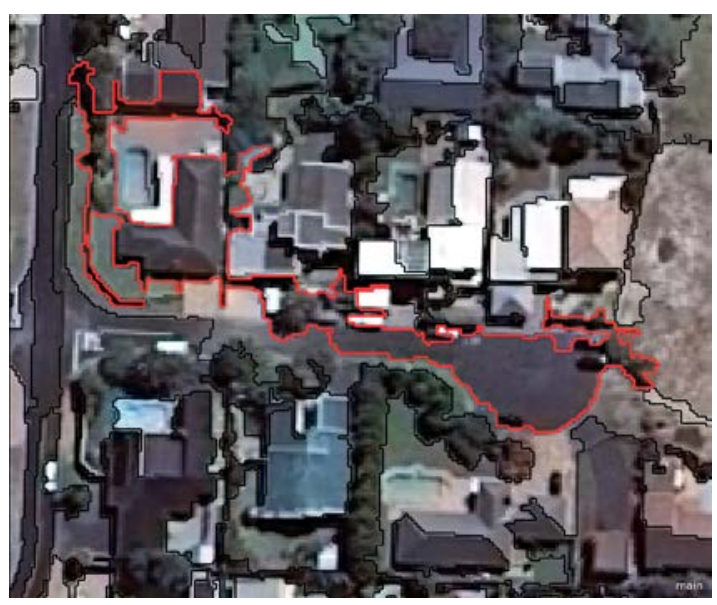

Figure 5c: Image segmentation (Level 1, scale: 100)

The classes created were built-up area, road (for identifying the larger roads that were not included in the built-up area), bare ground or sand, vegetation and water. Samples were selected for each class, and the image was classified using the Nearest Neighbour (NN) method. A large scale parameter was chosen for classifying the image in order to adequately represent the large built-up areas. The following features were used in the $\mathrm{NN}$ classification: mean values for red, green, blue and near infrared, brightness, maximum difference, compactness, length, length/width, HSI transformation, NDVI, GLCM mean (quick 8/11) (all dir.) and GLDV Ang. $2^{\text {nd }}$ moment mean (quick 8/11) (all dir.).

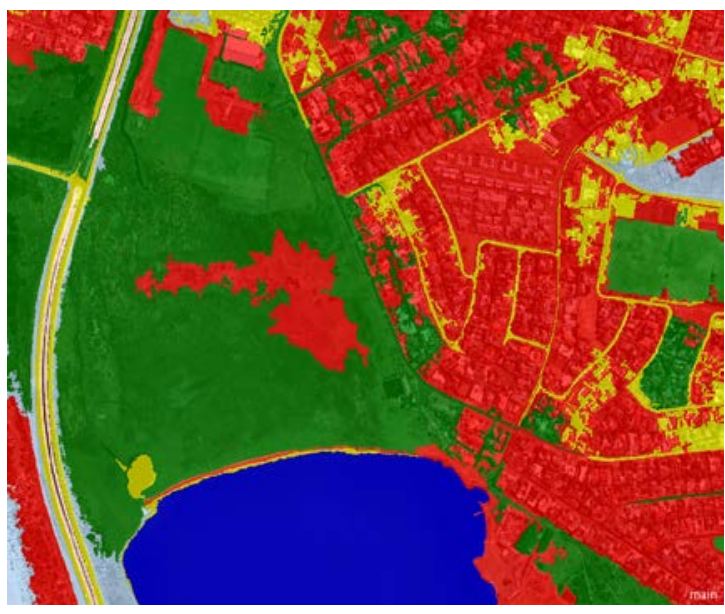

Figure 6: Object-based classification using the Nearest Neighbour method

The classification results indicate that there is some confusion between certain classes, particularly where there are segments that contain more than one feature. This is a typical problem where large segments contain more than one feature class. On the contrary, smaller segments may represent individual features more easily, but the spectral differences within classes may result in the user having numerous sub-classes for features.

\begin{tabular}{|l|c|c|c|}
\hline Class name & $\begin{array}{l}\text { Producers } \\
\text { accuracy }\end{array}$ & $\begin{array}{l}\text { Users } \\
\text { accuracy }\end{array}$ & $\begin{array}{l}\text { KIA per } \\
\text { class }\end{array}$ \\
\hline Built-up & 0.57 & 0.80 & 0.48 \\
\hline Road & 1.00 & 1.00 & 1.00 \\
\hline Vegetation & 0.71 & 0.56 & 0.59 \\
\hline Water & 1.00 & 1.00 & 1.00 \\
\hline $\begin{array}{l}\text { Bare ground or } \\
\text { sand }\end{array}$ & 0.50 & 0.50 & 0.46 \\
\hline Overall accuracy & \multicolumn{2}{|c|}{$\mathbf{0 . 7 9}$} \\
\hline KIA & $\mathbf{0 . 7 3}$ \\
\hline
\end{tabular}

Table 2: Accuracy assessment and kappa statistics for object-based classification (segments based on only spectral information)

\section{Using vector data and spectral information for segmentation}

In order to overcome the problem of having unsuitable segments such as those that spanned across roads or that contained mixed classes, vector data was included in order to segment the image based on cadastral information (information maintained and supplied by the Office of the Chief Surveyor General in South Africa). The initial segmentation was performed using the thematic layer to create segments at the cadastral layer level. 


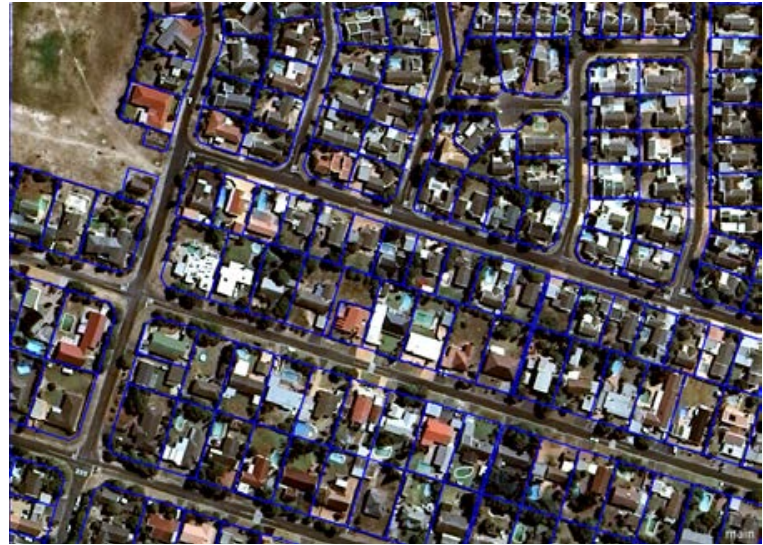

Figure 7: Segmentation based on cadastral parcels

After the initial segmentation, a second segmentation was performed within the boundaries of the cadastral segments. These resulting smaller segments were then classified using the nearest neighbour approach. Samples were selected and the following features were used in the NN classification: mean values for red, green, blue and near infrared, brightness, maximum difference and NDVI.

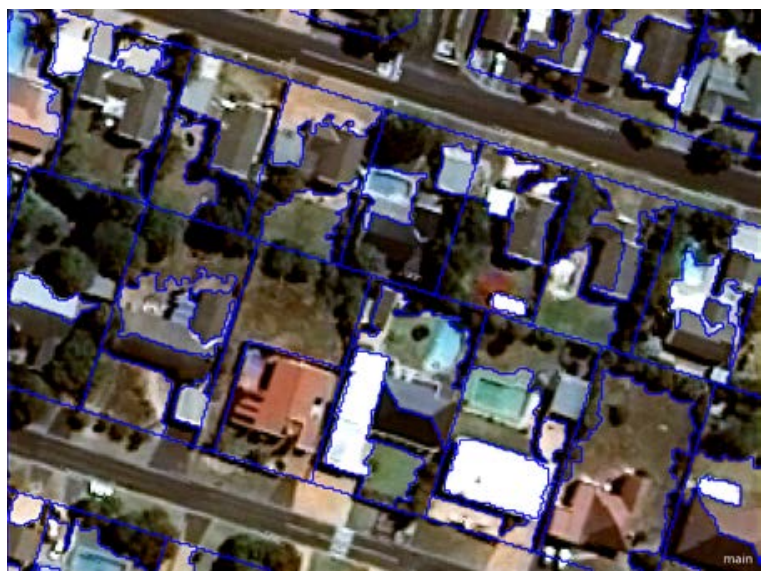

Figure 8: Segmentation within cadastral parcels

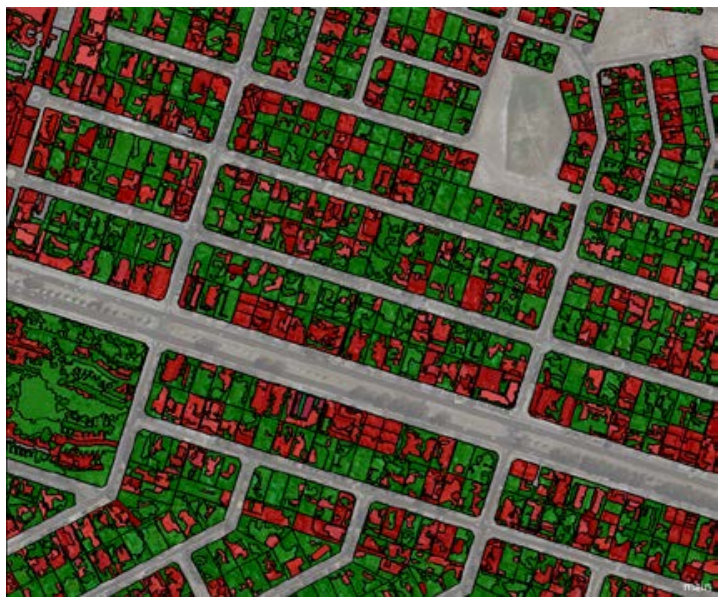

Figure 9: Object-based classification using the Nearest Neighbour method (initial segments derived from thematic information)

\begin{tabular}{|l|c|c|c|}
\hline Class name & $\begin{array}{l}\text { Producers } \\
\text { accuracy }\end{array}$ & $\begin{array}{l}\text { Users } \\
\text { accuracy }\end{array}$ & $\begin{array}{l}\text { KIA per } \\
\text { class }\end{array}$ \\
\hline Building & 0.94 & 0.89 & 0.90 \\
\hline Vegetation & 0.91 & 0.87 & 0.81 \\
\hline Water & 0.60 & 1.00 & 0.57 \\
\hline Overall accuracy & \multicolumn{2}{|c|}{$\mathbf{0 . 8 9}$} \\
\hline KIA & \multicolumn{2}{|c|}{$\mathbf{0 . 8 0}$} \\
\hline
\end{tabular}

Table 3: Accuracy assessment and kappa statistics for object-based classification (segments initially based on thematic information)

For this example only buildings, vegetation and water were classified and the areas between the cadastral blocks, for example large roads, were masked out of the classification (see Figure 9).

\section{DISCUSSION}

It is necessary to move away from dependency on individual pixel values into a way of incorporating shape, texture and contextual information for image classification (Hurskainen \& Pellikka 2004).

Segmentation is a very important step in object-based classification. In order to have a successful classification, one must have suitable segments that accurately represent features of interest. Segmentation based purely on spectral information did not result in suitable segments. The inclusion of thematic or vector data for the initial segmentation in the object-based classification resulted in an improvement in overall accuracy when compared with the method that was based only on spectral information.

It should be noted that the selection of evaluation or check sites has a large influence on the accuracy results reported. All check sites were randomly selected and were not part of the classification training sites. Since the accuracy assessments are always based on a sample of the classified scene, it is difficult to get a 'true' accuracy assessment.

\section{CONCLUSIONS}

The object-based classification methods show much promise and results in better classification accuracy than the pixel-based methods that were tested. The inclusion of thematic data in the segmentation stage can be used to force suitable boundaries that can be further segmented and thus improve classification results.

The decision regarding whether to classify individual buildings or larger built-up areas is an important factor to consider. Each option has its own merits and drawbacks. Individual buildings may be easier to detect based on their shape properties, but may vary greatly in spectral characteristics due to roofing types and materials used. One may need to have sub classes within the building class to adequately represent all building types. On the other hand, large built-up areas may be less homogeneous due to the inclusion of a variety of individual features within the built-up area for example, buildings, grass, trees, roads, etc. and therefore may be difficult to identify adequately and consistently. 


\section{REFERENCES}

Blaschke, T. et al., 2000. Object-oriented image processing in an integrated GIS/remote sensing environment and perspectives for environmental applications. Environmental information for planning, politics and the public, 2, pp.555-570.

De Dapper, M., Gamanya, R. \& De Maeyer, P., 2006 PRECISON CHANGE DETECTION: BASED ON KNOWLEDGE-BASED AND OBJECTORIENTED SATELLITE IMAGE ANALYSIS IN CENTRAL ZIMBABWE. In American Society for Photogrammetry and Remote Sensing (ASPRS).

Hofmann, P., 2001. Detecting urban features from IKONOS data using an object-oriented approach. In First Annual Conference of the Remote Sensing \& Photogrammetry Society, 12-14 September 2001. Munich, Germany, pp. 79-91.

Hurskainen, P. \& Pellikka, P., 2004. Change detection of informal settlements using multi-temporal aerial photographs: the case of Voi, SE Kenya. In Proceedings of the 5th AARSE conference (African Association of Remote Sensing of the Environment). pp. 17-22.

Lillesand, T.M., Kiefer, R.W. \& Chipman, J.W., 2004. Remote Sensing and Image Interpretation 5th ed., Wiley and Sons.

Walter, V. \& Fritsch, D., 1998. Automatic verification of GIS data using high resolution multispectral data. International Archives of Photogrammetry and Remote Sensing, XXXII(Part 3/1), pp.485 - 489.

Zhang, Y., 2001. Texture-Integrated Classification of Urban Treed Areas in High-Resolution ColorInfrared Imagery. Photogrammetric Engineering \& Remote Sensing, 67(12), pp.1359-1365. 